\title{
Arborescences
}

Revue d'études françaises

\section{Les communistes français et la promotion des langues régionales dans la période avant et après la Seconde Guerre mondiale}

\section{Olivier Moliner}

Numéro 1, mars 2011

Identités linguistiques, langues identitaires : à la croisée du prescriptivisme et du patriotisme

URI : https://id.erudit.org/iderudit/1001944ar

DOI : https://doi.org/10.7202/1001944ar

Aller au sommaire du numéro

Éditeur(s)

Département d'études françaises, Université de Toronto

ISSN

1925-5357 (numérique)

Découvrir la revue

Citer cet article

Moliner, O. (2011). Les communistes français et la promotion des langues régionales dans la période avant et après la Seconde Guerre mondiale. Arborescences, (1), 0-0. https://doi.org/10.7202/1001944ar
Résumé de l'article

L'article se propose de retracer les origines du long débat parlementaire en matière de politique linguistique en France qui a finalement débouché sur une reconnaissance officielle du patrimoine linguistique français et ce, surtout selon les aspects qui touchent à l'engagement des communistes français concernant le dossier parlementaire des langues régionales et leur introduction dans les différents degrés de l'Éducation nationale. Parmi les motifs à être éclairés sont les motifs politiques, les traits les plus marquants de cet engagement et le rôle joué par les communistes au sein de l'Assemblée nationale pendant la période qui précède la Seconde Guerre mondiale et à la période de l'immédiat après-guerre. L'auteur conclut que les communistes français étaient confrontés à la difficulté d'harmoniser trois positions différentes : le patriotisme pour une République unie avec le français comme langue nationale, la conception marxiste-stalinienne et l'attachement aux langues régionales. Pour atteindre ce triple objectif, les députés communistes se présentèrent comme une force patriotique marquée par la résistance au nazisme et au franquisme.
Tous droits réservés @ Département d'études françaises, Université de Toronto, 2011
Cedocument est protégé par la loi sur le droit d'auteur. L'utilisation des services d’Érudit (y compris la reproduction) est assujettie à sa politique d'utilisation que vous pouvez consulter en ligne.

https://apropos.erudit.org/fr/usagers/politique-dutilisation/ 


\title{
LES COMMUNISTES FRANÇAIS ET
}

\section{LA PROMOTION DES LANGUES RÉGIONALES}

\section{Dans la période avant et après la Seconde Guerre Mondiale}

\author{
Olivier Moliner \\ Université Libre de Berlin
}

\section{Introduction}

Compte tenu des récentes évolutions en matière de politique linguistique en France - depuis juillet 2008 les langues régionales telles que le breton ou l'occitan sont inscrites dans la constitution républicaine - il semble utile de retracer les origines de ce long débat parlementaire qui a finalement débouché sur une reconnaissance officielle du patrimoine linguistique français. Dans cette optique nous privilégierons surtout les aspects qui touchent à l'engagement des communistes français concernant le dossier parlementaire des langues régionales en général et leur introduction dans les différents degrés de l'Éducation nationale par des initiatives concrètes. Nous tenterons d'éclairer les motifs politiques, les traits les plus marquants de cet engagement linguistique et le rôle joué par les communistes au sein de l'Assemblée nationale (AN) tout en tentant de combler quelques lacunes qui subsistent sur le champ de la recherche. Nous nous limiterons à la période qui précède la Seconde Guerre mondiale de 1936 à 1939, date à laquelle le Parti communiste français (PCF) commença à s’intéresser aux langues régionales au niveau parlementaire et à la période de l'immédiat après-guerre de 1945 à 1952, espace de temps plus ou moins boudée par la recherche, mais étant particulièrement riche à notre égard. Nous passerons sous silence les années de la Seconde Guerre mondiale en France, époque durant laquelle le travail du parlement fut interrompu. Nous évoquerons en passant la genèse de la loi Deixonne (1951), première loi-cadre après la guerre offrant un statut scolaire exclusivement au breton, occitan, catalan et au basque. Une loi apparemment attribuée au socialiste Maurice Deixonne, mais qui fut initialement préparée par des députés communistes bretons, une loi qui avait au premier coup d'œil l'atout de traiter pour la première fois de la question des langues régionales de manière "presque» collective. «Presque », car l'alsacien, le flamand, le corse, les langues non-territorialisés et les langues des départements d'outre-mer furent ignorés voire écartés. Sans trop nous attarder sur le cheminement de la loi et sur son caractère collectif douteux, nous 
nous pencherons plutôt sur quelques positionnements, parfois contradictoires, du PCF envers la promotion des langues régionales, tel que l'alsacien, le catalan, le breton et l'occitan.

Nous interrogerons surtout les deux points suivants: Quels sont les argumentaires, les modèles politiques ou les réflexions auxquels les communistes se sont référés pour s'engager en faveur des langues régionales? Comment sont-ils parvenus à synchroniser patriotisme français, passé résistant, fidélité à la ligne du parti et engagement pour une diversité linguistique en France ? Notre texte n'a pas pour ambition de traiter le dossier de manière exhaustive ${ }^{1}$. L'article qui va suivre se propose d'élargir et d'approfondir partiellement le champ des études historiques. Un champ composé d'études régionales, qui a déjà été travaillé auparavant par quelques auteurs, soit de manière plus générale, incluant plusieurs langues régionales, comme chez Guiomar et Giacomo avec l'accent mis sur le breton, soit sous l'angle d'une langue particulière, comme l'occitan chez Lafont, Sagnes, Martel, Abrate et l'alsacien/l'allemand chez Reimeringer et Maugué.

L'article se divise en quatre parties. Dans la première partie, nous nous concentrerons sur la période précédant la Seconde Guerre (1925-1939) et nous nous pencherons sur la loi Dahlet (1936), qui jusqu'à là n'avait pas encore fait l'objet d'une étude, et sur le rapport Desgranges (1937), mieux connu. Dans la deuxième partie, couvrant les années 1945 à 1952, les députés bretons à l'avant-garde et le rôle de la Résistance chez certains communistes constitueront le cœur de notre étude. Dans la troisième partie, nous analyserons à partir du cas de l'Alsace et de la Lorraine le renouement tardif avec la politique du PCF de l'avant-guerre. Dans la dernière partie du texte, nous mettrons en lumière les traitements inégaux et plus ou moins arbitraires au sein du PCF à l'aide d'une comparaison des dossiers alsaciens, catalans et occitans.

\section{La préhistoire : de la Révolution à la Troisième République}

Contrairement à ce que l'on pourrait croire aujourd'hui avec l'introduction controversée ${ }^{2}$ des langues régionales dans la Constitution, le parlement français a longtemps rejeté avec fermeté toutes sortes de mesures tendant à promouvoir les langues régionales. Nous trouvons l’idéologie servant de support à ce rejet dans un discours plutôt langagier-nationaliste (Trabant 1981 : 71-72) chez Bertrand

\footnotetext{
${ }^{1}$ Nous renvoyons le lecteur à la thèse qui a été déposée par l'auteur. Cette thèse comprend des détails supplémentaires en ce qui concerne la politique des langues du PCF et des notes biographiques plus détaillées à l'égard de plusieurs députés communistes. Voir Moliner (2010).

2 Concernant la discussion au sujet de la ratification de la Charte européenne des langues régionales et minoritaires en France, voir Lebsanft (2004 : 175-188). Pour un résumé des débats parlementaires autour de la réforme constitutionnelle de 2008 voir Moliner (2010 : 13-16).
} 
Barère (1794) qui exalte «la prééminence de la langue française » (Certeau 1975 : 297) au niveau international. Le reflet de la pensée Barèrienne réapparaîtra ultérieurement dans les discours portant sur le rayonnement de la langue française à travers le monde chez Pompidou, Chirac et dans les débats autour de la loi Bas-Lauriol de 1975 (Trabant 1981: 71-72). Au niveau national, le conventionnel Barère affirme que « la langue d'un peuple libre doit être une et la même pour tous » (Certeau 1975 : 297) et il qualifie le bas-breton d'« instrument barbare » (Certeau 1975 : 292), tout en exemptant les dialectes d'oïl et l'occitan, car «ils n'ont pas empêché de connaître la langue nationale » (Certeau 1975 : 292). Nous trouvons le second pilier de cette idéologie dans le rapport de l'Abbé Henri Grégoire (1794) qui préconisa, sans admettre d'exception comme Barère, l'utilité d'anéantir les "patois» car les langues régionales se présentaient comme «un obstacle à la propagation des lumières » (Certeau 1975 : 304).

Les retombés idéologiques de ces deux rapports et la liaison étroite et ancienne entre État et langue (Cerquiglini 2000 : 600), qui fut complétée par l'élément nation pendant la Révolution, ont longtemps verrouillé (Martel 2007: 35) les esprits parmi les générations de parlementaires. Les premières initiatives qui tentèrent de surmonter les barrières idéologiques et de mettre en avant les droits des «langues de France» de manière collective surgirent avec la Pétition pour les langues provinciales en 1870. Ce document, qui fut rédigé par deux royalistes attitrés, le bascologue Hyacinthe de Charencey et le celtisant Charles de Gaulle, et par un républicain modéré, le celtologue Henri Gaidoz, deviendra la source de référence et l'argumentaire principal des défenseurs des langues régionales au parlement de la III $^{\mathrm{e}}$ République. Le premier succès ${ }^{3}$ limité allant dans le sens d'un enseignement facultatif de certaines langues régionales dans les trois degrés de l'enseignement sera adopté en 1951 avec la loi Deixonne. Bien entendu, le débat sur les langues régionales est beaucoup plus ancien que l'existence du parlement et des différents groupes parlementaires, dont celui des communistes. Pour la période allant de la monarchie capétienne ${ }^{4}$ jusqu'au Second empire, il nous faudrait creuser de manière bien plus profonde pour rendre visible les traits de la question des langues. Nous nous bornerons ici strictement au cadre parlementaire pour observer le cheminement du débat au sein du groupe communiste dans une période de temps limitée (de 1936 à 1952).

\footnotetext{
${ }^{3}$ Cependant il est vrai aussi que la loi Deixonne concernant les trois degrés de l'enseignement fut précédée en 1945 par la circulaire Monod pour le second degré, en 1941 par l'arrêté Carcopino concernant le primaire sous Vichy et par la circulaire François-Albert (1924) pour le second degré et les écoles normales dans le Midi sous la III e République (Moliner 2010 : 75-143).

${ }_{4}^{4}$ Dans ce contexte, voir l'ouvrage historique récent de Jean-François Courouau (2008), qui cerne systématiquement le champ des littératures régionales.
} 


\section{- Les premières initiatives de la gauche parlementaire}

Toutefois, il est vrai que, à partir de 1900, les premiers défenseurs des «langues provinciales» furent majoritairement des notables et des personnalités de tendance royaliste, catholique, d'extrême droite et conservatrice. A partir des années vingt, cette domination sera bientôt atténuée par des députés de sensibilité républicaine et de gauche, comme par exemple les républicains André Lefèvre (Bouches-du-Rhône) et Paul Guieysse (Morbihan), le chrétiendémocrate Pierre Trémintin (Finistère) ou le radical Hippolyte Ducos (Haute-Garonne). L'absence de citation du député Jean Jaurès, qui à partir de 1911 s'était déclaré en faveur d'un enseignement des langues régionales à l'aide d'une méthode comparée, ne cherche pas à occulter son engagement linguistique, bien que ce dernier fût plutôt d'ordre journalistique que parlementaire. L'absence souligne aussi le fait que Jaurès ne se soit jamais prononcé ouvertement au sujet des langues au sein de l'Hémicycle.

Une des caractéristiques des députés communistes qui se succéderont au Palais Bourbon après le congrès de Tours en 1920 sera de mener une politique linguistique collective et favorable à un enseignement dans les écoles, c'est-à-dire avec le consentement de la direction du parti. À deux reprises (en 1936 et en 1937), le groupe communiste votera ensemble avec des adversaires politiques (radicaux et conservateurs) pour faire passer des mesures favorables à l'égard des langues régionales. Cependant, ces deux premières prises de positions parlementaires visibles de la part de la Section Française de l'Internationale Communiste (SFIC) - elle prendra le nom de Parti Communiste Français en 1922 - ne furent pas le fruit d'un hasard, mais étaient liées à une réflexion et à une politique d'ensemble du parti. D'après Guiomar (1970), Reimeringer (1977) et Martel (2001), le PCF poursuivit à partir de 1925 et jusqu'en 1936 une politique anti-impérialiste avec l'option d'une autonomie comprenant la possibilité de la séparation des régions d'Alsace et de Lorraine de la France (Reimeringer $1977: 376)^{5}$.

\section{- Le dilemme des idéologies}

Il est évident que cette politique communiste, très calquée sur le modèle soviétique construit sur les définitions de la nationalité de Lénine et Staline $(1913)^{6}$, était doublement en contradiction

\footnotetext{
5 Maurice Thorez parle en 1932 du «droit à la libre disposition du peuple alsacien-lorrain jusque et y compris la séparation d'avec la France » (Maugué 1970 : 78).

${ }^{6}$ Il est question ici du texte "Marxisme et question nationale », rédigé par Joseph Staline en 1912-13. Il n'est pas clair à partir de quelle date et par quelle traduction le texte fut connu en France par les militants. A la Bibliothèque Nationale de
} 
avec l'idée française de la nation : à l'échelle politique, depuis 1789, la nation française était conçue comme une construction volontaire, un « plébiscite de tous les jours » pour citer Renan. D'après lui, « il y a dans l'homme quelque chose de supérieur à la langue : c'est la volonté » pour citer son fameux discours à la Sorbonne de 1882 (Forest 1991 : 38). Seconde différence, en France la langue nationale n'était pas un fait de masse comme le prescrivait la conception soviétique : la majorité des Français parlait le français seulement depuis la fin de la Première Guerre mondiale. Le français en tant que langue nationale était donc plutôt lié à un «fait administratif » émanant de la vieille liaison entre l'état et la langue française, pour citer Calvet (1974: 170). Tôt ou tard, le dilemme entre modèle soviétique et réalité française devait se manifester chez les communistes français. En attendant, le système soviétique était séduisant pour les libertés constitutionnelles accordées aux multiples minorités nationales en URSS, mais tout cela à la seule condition, selon le dogme stalinien, que les populations concernées puissent se définir en tant que communauté de culture, historiquement stable, constituée par la langue, le territoire, la vie économique et par la formation psychique. De même, la politique pratiquée en URSS semblait aussi avoir l'avantage d'une moindre division des travailleurs et des paysans.

\section{- Des répercussions concrètes sur le terrain}

Cependant, en France, la scission ${ }^{7}$ régionale du PC en Alsace et Lorraine de 1929 brisa très vite le rêve de l'unité fraternelle entre les militants du même parti. En 1936, avec la montée de la menace nazie, le PC fut aussi contraint de changer de cap pour une politique nationale et patriotique à la française. Ainsi le membre du PC alsacien Marcel Rosenblatt proclama devant le congrès national du PC en 1937 à Arles ${ }^{8}$ que « la garantie du salut du peuple d'Alsace réside uniquement dans son union avec celui de la France » (Rosenblatt cité dans Reimeringer 1977 : 385). Rosenblatt, futur député communiste du Bas-Rhin de l'après-guerre, sera en 1950 le rapporteur, avec la députée Maria Schell (Moselle), d'une loi et d'une résolution tendant à rétablir le bilinguisme dans les départements Haut-Rhin, Bas-Rhin et Moselle. À partir de l'année 1935, il semble que le PC se soit plutôt tourné vers un régionalisme occitan et breton, moins agressif et plus compatible avec les intérêts nationaux.

France nous avons trouvé le texte suivant, qui date de 1927 : Le Communisme et la question nationale et coloniale, par Lénine, Staline et Boukharine, Paris : Bureau d'éditions, de diffusion et de publicité : $64 \mathrm{p}$.

${ }^{7}$ La scission régionale du PC de 1929 fut provoquée par des communistes oppositionnels qui voulaient s'allier lors des élections aux forces autonomistes et séparatistes en Alsace et Lorraine (Reimeringer 1977 : 376 ).

${ }^{8}$ Martel (2001 : 373) décrit une scène folkloriste organisée à l'attention des participants du congrès du PC à Arles avec un défilé de gardians camarguais conduit par le marquis et félibre Folco de Baroncelli-Javon, lié amicalement à des communistes locaux. Guiomar (1970) rapporte qu'en décembre 1937, à l'aube du congrès arlésien, le PC rendit hommage à Frédéric Mistral en publiant une brochure intitulée Le parti communiste et les traditions du peuple français. 
Il est vrai que pendant la période du Front populaire, on trouvera au niveau régional, par exemple dans le journal Rouge-Midi (Martel 2001 : 373), plusieurs prises de positions favorables rédigées en occitan, mais également des opinions positives en vue d'une prochaine promotion de l'occitan. Barel, candidat communiste aux élections législatives et porte-parole des autres candidats du PC en Alpes-Maritimes, affirmait à ce sujet dans Le Feu, organe du régionalisme méditerranéen : « [...] des alphabets ont été créés pour certaines régions. Nous sommes décidés, quant à nous, sans arrière pensée aucune à soutenir toute mesure en faveur des langues particulières » (Guiomar 1970 : 105). En somme, les véritables résultats parlementaires revendiqués maintes fois par les candidats du PC lors de la campagne électorale pour aider à faire entrer l'occitan dans les écoles, resteront complètement invisibles à la Chambre jusqu'au déclenchement de la Seconde Guerre mondiale.

\section{- De la loi Dahlet (1936) à l'aube de la Seconde guerre mondiale}

Même si le PC avait adopté une ligne nationale en abandonnant les idées séparatistes, cela ne l'empêcha pas de poursuivre son engagement linguistique: les principaux bénéficiaires de cette politique du PC, qui finalement se soldera par un échec parlementaire pour les langues en question, furent le breton (avec le rapport Desgranges en 1937) et l'alsacien/allemand (avec la loi Dahlet en 1936). Il semble que l'occitan ne joua plus aucun rôle dans les activités parlementaires du PC à partir de cette époque. La loi du 30 juillet 1936, nommée d’après le député radical Camille Dahlet du BasRhin, «tendant à introduire le bilinguisme intégral»(Loi Dahlet [LD] 1936 : 1560-1561) dans les écoles, la justice et la presse des départements Bas-Rhin, Haut-Rhin et Moselle fut soutenue par une alliance de députés de tous bords. Parmi les signataires figurent des noms comme Robert Schuman et Anatole de Monzie - fait curieux, car l'ancien Ministre de l'Éducation nationale avait rédigé une circulaire en 1925 très hostile à «la renaissance des patois » (Monzie 1925 : 210) dans les écoles publiques. Pierre Trémintin (député qui refusera les pleins pouvoirs à Pétain en 1939 et qui fut l'auteur d'un premier projet de loi en faveur du breton en 1936, qui finalement aboutira dans le rapport Desgranges), Jacques Doriot (ex-communiste qui deviendra un des appuis de la collaboration avec les nazis), Émile Béron (PC-oppositionnel) et Charles Hueber (PC-oppositionnel, maire de Strasbourg, partisan de l'autonomisme) furent eux aussi signataires. Les élus alsaciens au sein du groupe parlementaire du PC, fort décimés après la scission régionale de 1929, sont représentés par le seul député «ligniste » Alfred Daul (Bas-Rhin), qui fut secondé par le numéro un du parti national Maurice Thorez (Seine). 
Le projet Dahlet ne cache pas sa critique du gouvernement porté alors par le Front populaire qui l'accusait de négliger les droits linguistiques des deux-tiers de la population alsacienne et d'exercer une «politique d'assimilation précipitée et forcée » (LD 1936 : 1560-1561). Quoi qu’il en soit, le projet n'aura pas de suite et la trace de la loi se perdit dans les rouages de la Commission de l'Alsace et Lorraine. Le rapport Desgranges de 1937, second projet parlementaire, fut lui aussi finalement adopté avec l'aide des députés communistes. Ce dernier texte fut rédigé par l'abbé Desgranges, député d'orientation catholique et sociale de Vannes, qui eut le soin de rapporter la loi Trémintin de 1936, loi en faveur du breton «tendant à introduire le bilinguisme » (Loi Trémintin [LT] 1936 : 768) dans l'enseignement public des départements bretonnants. On peut considérer Pierre Trémintin comme le vétéran parlementaire du breton: élu de Plouescat (Finistère) et bretonnant, il s'était déjà prononcé en 1924 et 1925 à la tribune du parlement pour critiquer la circulaire Monzie de 1925. Dans ce contexte, le rapporteur Desgranges prit le temps de rédiger un texte abondant et détaillé (résumé de l'histoire du breton, données sur les locuteurs, sur la littérature, sur le mouvement de défense du breton, avec des références à Bebel, Masson, Sohier et Jaurès) tout en faisant découvrir aux parlementaires, probablement pour la première fois dans l'histoire de la Chambre, les aspects multiples et les richesses littéraires «de ce patrimoine national de la France» (RD 1937 : 917). Pour obtenir gain de cause, la Commission recommanda de transformer le projet Trémintin en proposition de résolution non-contraignante pour le gouvernement.

L'adoption finale de cette résolution désamorcée à la Commission de l'Éducation nationale réussit grâce au support du député communiste Darius le Corre (Seine-et-Oise) ${ }^{9}$, du radical Albert le Bail (Finistère) et du conservateur René Dommange (Seine). La mesure finale, qui invitait «à étudier [...] et à mettre en application l'enseignement de la langue bretonne, parallèlement à celui de la langue française » (RD 1937 : 919) dans les écoles primaires, collèges et lycées du Finistère, ne fait plus mention explicite de l'«option pour le breton comme seconde langue aux examens du baccalauréat» (LT 1936 : 768). La résolution se contente de proposer des mesures transitoires, de façon à gagner encore plus de temps. Au final, elle ne sera jamais appliquée, et tout cela en dépit des interventions personnelles adressées au ministre Jean Zay, notamment celles du député radical Albert le Bail (Finistère) (cf. Le Bail 1948 : 137) ${ }^{10}$, du socialiste François Tanguy-Prigent (Finistère) (cf. Keravel 1970 : 313) et du militant et poète Jòrgi Reboul (Marseille) (cf. Martel 2005 : 305).

\footnotetext{
${ }^{9}$ «Dire que je ne veux avoir rien de commun avec les Communistes? Mais ils ont appuyé avant-hier ma proposition sur l'enseignement du breton. » (Desgranges 1960 : 144)

${ }^{10}$ Voir aussi Moliner (2009 : 127).
} 


\section{Les députés bretons à l'avant-garde et le rôle de la Résistance chez certains communistes}

Dès 1947, les députés bretons communistes des trois départements bretonnants (Finistère, Morbihan et Côtes-du-Nord) présentèrent une première proposition de résolution «tendant à l'organisation d'un enseignement de la langue bretonne » (Résolution Hervé [RH] 1947 : 1) dans les trois degrés de l'enseignement public. La résolution Hervé réclame une formation pour les maîtres, l'autorisation du breton au baccalauréat avec un statut de seconde langue et la création d'un Institut d'études celtiques à Rennes. Il est question du breton en tant qu'« instrument d'une culture populaire » (RH 1947 : 3) qui a le mérite d'être pris en compte par l'école. Le nombre des locuteurs y est chiffré à un million de personnes. La résolution nommée d'après son auteur, le député communiste Pierre Hervé, avait pour objectif l'abrogation de tous les règlements scolaires interdisant l'usage du breton dans les écoles publiques. Les règlements en question dataient de l'époque de Jules Ferry $^{11}$. Quelques années auparavant, le Gouvernement provisoire avait annulé une grande partie des lois et décrets adoptés par le régime de Vichy, notamment la circulaire Ripert (1940) et l'arrêté Carcopino (1941). Supprimer les lacunes législatives était alors un des objectifs à la sortie de la Guerre.

Le second objectif d'Hervé était de réclamer un traitement égal du breton et de l'occitan car seul le «provençal » avait obtenu dans le cadre d'une mesure dérogatoire (circulaire Monod 1945) ${ }^{12}$ quelques droits limités (des cours facultatifs de provençal dans le second degré de l'Académie d'Aix). Ce fait est assez curieux car cette dernière mesure, qui rétablissait le status quo ante de la Troisième République, c'est-à-dire le retour à la circulaire François-Albert de $1924^{13}$, fut obtenu en pleine période de transition, tout en excluant les autres dialectes occitans et langues régionales. Par conséquent, à partir de 1945, la nécessité d'une loi équitable et collective devenait une revendication incontournable.

Le texte d’Hervé fut très influencé par la pensée d'Armand Keravel, un enseignant sympathisant du PC et porte-paroles d'Ar Falž, une association d'enseignants laïcs bien ancrée à gauche et fondée par Yann Sohier en 1933. Keravel connaissait bien les députés finistériens de

\footnotetext{
11 «Le français sera seul en usage dans les écoles », décret d'application du ministère de Jules Ferry (18 mars 1881) (Chanet $1996: 206)$.

12 Pour la retranscription de la circulaire Monod, voir Moliner (2010 : 326).

13 Cette circulaire du 24 décembre 1924 rédigée par le Ministre de l'Instruction de l'époque François-Albert n’a à ce jour pas pu être retrouvée. Un extrait publié se trouve dans Lougal (1925 : 68).
} 
l'après-guerre car il était leur principal conseiller linguistique. L'initiative émanait d'un groupe de députés régionaux, Gabriel Paul, Alain Signor, Marcel Hamon, Hélène Le Jeune, Louis Guiguen et Marcel Cachin ${ }^{14}$, majoritairement des locuteurs de breton ${ }^{15}$. Autre élément décisif, tous ces députés provenaient d'un milieu ouvrier et rural et ils avaient participé de manière active ou passive aux mouvements communistes de la Résistance de Basse-Bretagne. La résolution faisait d'ailleurs plusieurs fois référence aux différentes formations de résistance (Forces françaises de l'intérieur et Forces françaises libres) et elle rejetait tout reproche de collaboration ou de séparatisme.

Et dans cette guerre, la Bretagne a fourni des milliers de volontaires, tant aux F. F. L. qu'aux F. F. I. Les «séparatistes » à la solde de l'Allemagne ont été vomis par la Bretagne patriote. Dans la Bretagne bretonnante, la résistance s'est illustrée par son héroïsme. (RH 1947 : 4)

\section{- L'influence communiste dans les associations bretonnes à Paris}

Pierre Hervé qui fut invité en 1947 à donner une conférence au Foyer breton de Paris (KerVreiz) s'exprima de manière à ne laisser planer aucun doute concernant son engagement patriotique :

L'indépendance de la Bretagne fut une période très brève de son histoire. L'unité géographique du sol n'existe pas, ni l'unité de race. La langue est le fait breton le plus important, mais les limites linguistiques ne correspondent pas aux limites historiques [...] il existe donc une nation française, mais pas de nation bretonne [...] La cause de la France s'identifie avec la cause du progrès et de la liberté. Pas de fédéralisme français! (cité d'après Toulemont 1947b : 1)

Ce genre de déclaration montre que les communistes eurent alors fort besoin de manifester leur patriotisme en plus de leur passé résistant - le mauvais souvenir de la collaboration, l'action du Parti national breton (PNB) et l'activité linguistique-nationaliste d'un Roparz Hemon étaient encore trop présents. On note aussi que ce sont des militants, des universitaires et des députés communistes d’origine de Basse-Bretagne, tel que le linguiste François Falc'hun, Susanne Jaffrés, Pierre Hervé ou encore Marcel Cachin, qui, dès 1944, tentèrent d'accaparer les infrastructures culturelles bretonnes et non-chrétiennes situées à Paris. L'emprise communiste (Calvé 1994 : 231) sur le centre culturel Foyer breton de Paris (Ker-Vreiz), qui était présidé à cette époque par Léon Toulemont ${ }^{16}$, un important défenseur du breton et critique littéraire chez l'hebdomadaire La Bretagne à Paris, se poursuivit dès la

\footnotetext{
14 Marcel Cachin, désigné chez Lafont (1974 : 226) comme « ce vieil allié de l'autonomisme révolutionnaire » ne semble pas avoir participé à la rédaction, mais son rôle à Paris soit dans le groupe, soit dans les instances du parti, a sûrement joué.

15 À l'exception du communiste Brestois Gabriel Paul. Voir ci-dessous.

${ }^{16}$ Léon Toulemont (1896-1959), dont nous pensons que le rôle en tant que lobbyiste du breton a été sous-estimé par l'historiographie, jouera par la suite un rôle primordial dans le cadre de l'adoption de la Loi Deixonne. Voir Moliner (2010).
} 
Libération, principalement portée par des militants de l'association Bretons émancipés ${ }^{17}$. Cependant, cette mainmise se révéla de courte durée, les milieux conservateurs et nationalistes reprenant dès les années 60 le contrôle de ce lieu parisien. C’est aussi grâce aux militants communistes que des institutions culturelles telles que Ker-V reiz, furent autorisées et rouvertes par les autorités préfectorales dans l'immédiat après-guerre. Cette volonté communiste d'agir dans le sens d'une reconnaissance et d'une promotion de la langue bretonne s'accorde avec les activités de l'avantguerre, et nous soulignons par ailleurs que le PC avait déjà soutenu, vainement certes, le rapport Desgranges (1937).

\section{- Les députés communistes et leurs alliés au parlement}

Pareillement, en 1948, les sénateurs communistes n’hésitèrent pas non plus à conclure une nouvelle alliance avec divers partis de droite et de gauche afin de surmonter le blocage exercé au sein de la Commission de l'Éducation nationale par la députée Rachel Lempereur (Nord) de la Section française internationale ouvrière (SFIO) hostile à un possible «affaiblissement de la langue nationale » (Résolution Lempereur [RL] 1947 : 2). En juillet 1948, ce fut donc le sénateur finistérien et résistant décoré, Antoine Vourc'h, Rassemblement du peuple français (RPF), un membre du Mouvement républicain populaire (MRP) qui venait d'adhérer au mouvement gaulliste, qui déposa un projet de loi «Sur l'enseignement de la langue bretonne » (Loi Vourc'h [LV] 1948 : 1). Le projet fut cosigné par les sénateurs Yves Henry (SFIO/Côtes-du-Nord), Auguste Le Coent (PCF/Côtesdu-Nord) et Pierre Trémintin (MRP/Finistère). Bien que les signataires aient appartenu majoritairement au MRP, la contribution idéelle émanant du sénateur communiste Le Coent, originaire de Ruellu en Côtes-du-Nord, ressortait clairement dans le texte avec des citations de Yann Sohier, August Bebel ou encore avec un éloge exprimé à l'adresse de l’Union soviétique :

C'est la politique choisie par l'U.R.S.S.; c'est ce qui explique la rapide diffusion de l'instruction parmi les populations hier encore très arriérées, instruction qui est un élément appréciable de la force actuelle de l'U.R.S.S. (LV 1948 : 11)

Les textes Hervé (1947) et Vourc'h (1948) réaffirment que «la Bretagne bretonnante est incontestablement moins conservatrice que la Bretagne non-bretonnante»(LV 1948: 4). La résolution Hervé et la loi Vourc’h seront finalement incorporées dans la future loi-cadre préparée par le socialiste Maurice Deixonne en 1949.

17 L'organisation Bretons émancipés fut fondée par le communiste Marcel Cachin, un des cosignataires de la résolution Hervé de 1947 le 19 décembre 1931 à Paris. Quelques membres communistes des Bretons émancipés comme Suzanne Jaffrès ou comme le linguiste François Falc'hun (Rennes) siégeaient dans le premier comité directeur de Ker-Vreiz en février 1945. Voir Guiomar (1970 : 106) et Ar Falz (1946). 


\section{- Le fait de la Résistance chez les parlementaires bretons}

Il semble aussi que l'expérience de la Résistance ${ }^{18}$ ait joué un rôle important dans l'élaboration des deux textes. En somme nous retenons les points suivants: Les députés communistes, mais aussi ceux du MRP, avaient souvent en commun l'expérience de la guerre, le passé résistant, l'origine commune de Basse-Bretagne et le fait d'être issus d'un groupe de locuteurs ayant appris le breton par transmission familiale. Le groupe des sept communistes autour de Pierre Hervé était influencé par les militants d'Ar Falź favorables à une introduction scolaire du breton, mais il rejetait tout ce qui pouvait ressembler à un régime bilingue ou qui s'apparentait au fédéralisme. Hervé récusait ainsi l'idée d'une région économique de Bretagne et ceci lui permettait de rester bien ancré dans le camp d'une République française, détentrice d'une tradition jacobine. Il semble que la contradiction apparente avec le modèle soviétique, qui prévoyait une large promotion et l'autonomie des langues et nationalités minoritaires, ne gêna personne au sein du parti. Bien au contraire, on a l'impression que le PCF se méfiait d'une décentralisation trop hâtive et qu'il avait bien retenu la leçon de l'avant-guerre. C'est probablement avec ce genre de concept mixte qui s'accordait aussi bien au patriotisme français qu'au modèle soviétique que les députés bas-breton sont parvenus à convaincre le bureau national de soutenir les premières initiatives en faveur du breton au parlement. Il semble que ce succès découla aussi de la force de persuasion des militants locaux. Nous retrouvons quelques éléments de leur argumentaire dans le témoignage personnel de l'ancien député de Brest, Gabriel Paul, dernier témoin vivant et cosignataire de la résolution Hervé de 1947 :

Tous ont été membres de la Résistance, à ma connaissance, ils n'avaient aucun lien particulier à la langue bretonne. Mais cela ne nous empêchait pas d'être sensible au fait qu'un nombre important de Bretons était attaché à leur langue. [...]. Ce texte a été déposé avec l'accord de la direction du P. C. F. et du groupe communiste et apparentés. Personnellement, j'ai pris conscience de l'intérêt de la langue bretonne lorsque j'étais dans la clandestinité. J'ai donc quitté Brest et rejoint d'abord le Morbihan où j'ai été chargé de réorganiser le mouvement syndical puis de retour dans le Finistère, j'ai été, au sein du triangle départemental du P. C. F chargé de l'organisation des maquis des francs-tireurs et partisans et notamment des parachutages d'armes à la libération, j'étais membre du comité départemental de la libération. Du fait de mes responsabilités, j'étais souvent appelé à me déplacer. En ces circonstances j'ai été hébergé chez des résistants dont le breton était la langue usuelle. Et je dois dire qu'ils étaient souvent surpris que, Brestois, je ne sache pas un mot de breton. Aussi notre proposition de résolution était pour moi, fondée et un geste de reconnaissance à ceux qui m’avaient hébergé. (Paul 2007a, b)

18 Giacomo (1975 : 27) écrit à ce sujet : «Surtout, les projets de loi reflètent, notamment dans l'exposé des motifs, le climat d'union à la fois populaire et nationale qui avait animé la Résistance. Avec l'approfondissement du sentiment national et de la volonté de libération du pays dans les couches les plus larges de la population s'est développée la conviction que la diversité nationale n’est pas nécessairement génératrice de séparatisme [...]. ». 


\section{Alsace et Lorraine : tardif renouement avec la politique du PC de l'avant-guerre?}

Prenons maintenant en compte la politique du PCF concernant l'alsacien et l'allemand dans les départements Bas-Rhin, Haut-Rhin et Moselle dans l'après-guerre. En comparaison avec la situation de la langue bretonne à la même période, le PC s'engagea relativement tard pour la défense des dialectes alsaciens et lorrains et de la langue allemande. Ces réticences apparaissent logiques si l'on garde en mémoire que le PC «anti-impérialiste» d'avant guerre avait été contraint de changer brusquement de direction devant le danger hitlérien pour se tourner vers une politique « nationale et patriotique », comme nous le verrons plus tard. Du point de vue linguistique, à la sortie de la guerre, les dialectes franciques-mosellans et alémaniques étaient encore largement pratiqués par la majorité des populations d'Alsace et de Lorraine ${ }^{19}$. De même, la langue allemande restait la langue écrite pour une grande partie de la population. Le bilinguisme était un fait marquant: par exemple, le journal communiste L'Humanité d'Alsace-Lorraine continuait de paraitre en allemand et la presse écrite était souvent bilingue.

Dans un premier temps (de 1944 à 1950), le PC eut alors tendance à escamoter les revendications linguistiques pour ainsi laisser le champ libre aux chrétiens-conservateurs, représentés par le député Henri Meck (MRP/Bas-Rhin) et Jacques Fonlupt-Espéraber (MRP/Haut-Rhin) qui entreprirent sans succès d'introduire l'alsacien dans le cadre de la loi Deixonne. D'un point de vue politique, la question linguistique dans les départements recouvrés restait très délicate et gênante, ce qui s'explique aussi par la mémoire douloureuse de la dernière guerre. En mars 1950, c'est le numéro trois du PC, François Billoux, qui, dans les Cahiers du communisme, sauta le pas en invitant camarades et cadres du parti à parler en alsacien et à écrire en allemand pour soutenir "des revendications » et «des particularités nationales évidentes »(Billoux 1950b : 52) et se mettre ainsi en phase avec la masse des mineurs mosellans et des paysans de la région d'Alsace et de Lorraine. Dans la perspective d'un possible rapprochement franco-allemand dans le cadre d'une future alliance économique, Billoux appela à combattre l'idée d'une telle union "germano-française », de "marchands de canons » (Billoux 1950b : 54) et de créer dans les instances alsaciennes du parti « une direction liée

\footnotetext{
19 «La majorité des populations alsaciennes s'expriment en dialecte. Dans le département de la Moselle, exception faite des régions de langue française, la langue allemande est employée par plus de $90 \%$ des habitants du bassin houiller de Forbach et des régions agricoles de Bitche, Wolmunster, Sierch, etc. De même que les régions sidérurgiques de Hayange, Hagondange, Thionville, etc. comptent un pourcentage de personnes ne s'exprimant pas ou difficilement en langue française. » (Loi Rosenblatt/Schell [LRS] 1950 : 1)
} 
aux masses [...] pouvant s'exprimer dans la langue utilisée par les travailleurs » (Billoux 1950b : 57). En se référant à Lénine et Staline, Billoux renouvela la vieille idée d'un « droit à la libre disposition, jusqu'y compris à la séparation » et y ajouta que cela ne signifiait pas «l'obligation à la séparation », comparable en cela au droit de divorce qui ne correspond pas non plus à «l'obligation de divorcer» (Billoux 1950a : 43). L'année suivante, en 1951, Billoux réaffirmera les fondements d'une politique alsacienne renouvelée en saluant à nouveau la politique de Staline au sujet des minorités nationales :

[...] Nous avons devant nous les résultats heureux de la politique de l'Union Soviétique basée sur les principes marxistes-léninistes qui ont eu, sous la direction de Staline, leur confirmation éclatante tout en s'enrichissant. 1.) Reconnaissance des nations comme réalité historique concrète d'après la définition marxiste-stalinienne: communauté stable historiquement constituée, de la langue, de territoire, de vie économique, de formation psychique qui se traduit dans la communauté de culture. 2.) Droit des peuples à disposer d'eux-mêmes. Marx nous ayant appris qu'un peuple qui opprime un autre ne saurait être un peuple libre [...]. (Billoux $1951: 20$ )

Dès lors, l'année 1950 était porteuse des premiers fruits parlementaires de ce renouement partiel avec la politique de l'avant guerre : tous deux persécutés par les nazis pour leurs idées politiques, les députés communistes bilingues Marcel Rosenblatt (Bas-Rhin) et Anne-Marie Schell (Moselle) déposèrent une première proposition de résolution en juin 1950 qui visait à « remettre en pratique les dispositions règlementaires et administratives relativement à l'enseignement de la langue allemande » (RRS 1950 : 870). La résolution communiste revendiquait le retour aux règlements et dispositions linguistiques, par exemple à la circulaire Pfister du 31 janvier 1927 qui avait été en vigueur dans l'entre-deux-guerres pour l'enseignement dans les trois départements d'Alsace et Lorraine. La circulaire de Christian Pfister, désignée par Maugué (1970 : 49f.) comme la « charte du bilinguisme scolaire », garantissait dans le primaire, à côté d'un apprentissage généralisé du français, un enseignement de l'allemand à partir du milieu de la deuxième année jusqu'au certificat d'études pour tous les enfants. La seconde initiative, la proposition de loi de Marcel Rosenblatt et d'AnneMarie Schell déposée elle aussi avec le consentement du groupe parlementaire du PCF en décembre 1950, élargit le cadre des revendications à l'ensemble de l'administration en proposant l'utilisation des deux langues (français/allemand) pour les administrations de l'État et celles des départements ainsi que celles des communes n'ayant «qu'un seul fonctionnaire» qui devra désormais «obligatoirement posséder les deux langues »(Loi Rosenblatt/Schell [LRS] 1950 : 3). Les deux initiatives parlementaires, quoique discutées au sein de la Commission de l'Éducation Nationale, resteront finalement sans effet. Sans vouloir présumer d'un lien direct, il se trouve que, deux années plus tard, le ministre de l'Éducation nationale André Marie publia le décret du 18 décembre 1952 « relatif à l'enseignement de la langue allemande dans les classes terminales des écoles primaires des 
communes dont la langue usuelle est le dialecte alsacien» (Nouvet 2001 : 23). Ce décret était en quelque sorte une compensation tardive pour l'exclusion, très critiquée, de l'alsacien et de l'allemand dans le cadre de la loi Deixonne en 1951.

En résumé, on peut dire que les communistes ne s'engagèrent que tardivement pour l'alsacien et l'allemand. Ce retard sera bientôt comblé par un activisme frappant pour la langue et les dialectes des mineurs et paysans d'Alsace et Lorraine. Néanmoins et en premier lieu, il nous faut replacer cet activisme pour un bilinguisme dans le cadre d'un débat politico-géo-stratégique sur une future région européenne, d'un «combinat Ruhr-Lorraine et Strasbourg » et d'un rapprochement avec l'«Allemagne revancharde» (Billoux 1950b: 54). En second lieu, la préparation de la loi Deixonne a certainement motivé les esprits. Le rapport Deixonne, qui fut soutenu par une majorité de tous bords politiques, le PC inclus, se contenta de promouvoir le breton, l'occitan, le basque et le catalan, tout en fermant injustement la porte à l'alsacien, au corse et au flamand. L'engagement tardif du PC pour les intérêts de l'alsacien exclu intervint alors à un moment, qui était bon et mauvais à la fois : mauvais, car à partir de 1948 le groupe du PCF s'était fait évincé pour l'obtention d'un poste de rapporteur concernant les projets Hervé et Vourc'h. Le socialiste Deixonne, allié à l'occasion avec le MRP, remporta l'élection à ce poste convoité au sein de la Commission de l'Éducation nationale au détriment des communistes. L'année suivante le députe Marcel Hamon (PCF/Côtes-du-Nord) fut contraint de remettre la fonction de rapporteur concernant la loi Marty aux mains de Maurice Deixonne, l'ennemi juré des communistes. Mais c'était aussi le «bon moment», car, presque démuni $^{20}$ de son engagement linguistique, le PC, qui pourtant avait été le précurseur au parlement, se dépêcha donc de revendiquer les mêmes droits pour l'alsacien et l'allemand, lésés par les autres partis, en mettant à jour la vieille revendication d'un régime bilingue, en plus de l'idée erronée d'un autonomisme alsacien, qui datait de l'avant-guerre et qui était, de nouveau, voué à l'échec devant la majorité des députés de l'AN.

\section{- La loi Marty, premier projet de loi en faveur du catalan}

La première initiative parlementaire en France pour une reconnaissance scolaire du catalan dans le département des Pyrénées-Orientales fut la loi Marty de 1948. Ce texte était étroitement lié

\footnotetext{
20 Pas tout-à-fait démuni, car le PC continua à militer au sein de la Commission, avec succès, pour faire avancer les intérêts du breton dans la nouvelle loi-cadre rapportée par Deixonne. Il faudrait mentionner ici surtout l'action du duo finistérien, Alain Signor ct le MPR André Monteil, qui présenta une proposition de loi supplémentaire et inédite, une synthèse entre la loi Vourc'h et la résolution Hervé. Le but de cette alliance entre «apparatchiks » et "curés » était l'introduction du breton dans le texte final.
} 
au personnage emblématique du communiste André Marty, originaire de Perpignan, maitrisant le catalan et apparenté au poète Antoine Cayrol alias Jordi Pere Cerdà. Marty, ancien mutin de la Mer Noire et vétéran de la guerre d'Espagne, avait aussi lutté aux côtés des républicains en Espagne. Ce dernier fut également longtemps député du département de la Seine et membre du bureau national du PCF avant d'en être exclu pour raisons politiques. Si l'on compare son projet avec l'initiative pour le breton d'Hervé, Marty revendiquait uniquement l'insertion facultative « de la langue catalane dans les Universités de Montpellier et Toulouse et dans certains établissements d'enseignements » (Loi Marty [LM] 1948: 1), laissant de côté le primaire, mais incluant par contre les écoles commerciales dans le département des Pyrénées-Orientales.

Le projet Marty s'engageait pour une prise en compte scolaire du catalan, une langue parlée des deux côtés des Pyrénées et qui disposait, d'après le texte, de 185000 catalanophones en France. De même, il préconisait une admission du catalan au baccalauréat. En insistant sur l'ancienneté de l'écriture catalane, sur le passé révolutionnaire pendant lequel les populations catalanes du nord, désireuses de libertés citoyennes, avaient lutté aux côtés des armées révolutionnaires contre l'aristocratie régionale pour finalement se fondre librement dans la nation et dans la langue française, Marty s'affirmait aussi en tant que patriote fidèle à la France : «Il n'y a plus aujourd'hui de minorité nationale catalane, il n’y a plus que des Français » (Marty 1952 : sans pages). Par contre, il saluait les aspirations républicaines et nationalistes de l'autre côté de la frontière, en Catalogne du sud car, pour Marty, c'est tout un peuple avec sa propre nationalité catalane qui s'était élevé contre l'oppression franquiste. En somme, pour lui, l'enseignement du catalan correspondait premièrement à une leçon « de la démocratie en France » et deuxièmement à un geste fort de solidarité avec la langue «d'un peuple frère » (Marty 1952 : sans pages), une langue qui «a aidé à la lutte contre le fascisme » ${ }^{21}$ et qui avait déjà acquis le statut de langue officielle de 1931 à 1939 en Catalogne.

\section{- Catalan et occitan : Deux traitements inégaux}

Autre fait curieux dans ce même contexte : dans un article du Patriote de Toulouse du 16 février 1952, Marty s'attaqua à l'occitan ${ }^{22}$. Il le considérait comme inférieur au catalan par son manque de prestige et émettait des doutes sur l'unité de l'occitan, une langue qui était, d'après lui, trop divisée en dialectes, et, fait plus important encore selon lui, les Occitans ne disposaient pas de nationalité

\footnotetext{
21 Nouveau titre d'une chanson traditionelle catalane «Mountanyas regaladas » du Roussillon et de la Cerdagne, dont les paroles furent réécrites par Marty pendant la guerre. Voir Cadé (1998 : 69-70).

22 Cet article a été signalé la première fois par Félix Castan à Philippe Martel (1990 : 56). Nous remercions beaucoup Yan Lespoux, qui l'a finalement retrouvé dans les archives personnelles de Robert Lafont et qui nous l'a communiqué.
} 
propre, le Languedoc et la Provence ayant été très tôt rattachés à la Couronne capétienne. Par conséquent, d'après lui, il en résultait un manque de sentiment d'appartenance nationale. Marty justifiait son dédain pour la langue d'oc en faisant référence à la définition de nation et de nationalité qu'en donna Staline en 1913 dans son texte Marxisme et question nationale, texte réédité et recommandé à la lecture pour les militants par le PC à partir de 1945 en France. Cette réflexion théorique au sujet des minorités nationales nous parait très caractéristique de la politique linguistique du PCF. Un des dirigeants, François Billoux, la réitéra en 1951 : «communauté stable historiquement constituée, de la langue, de territoire, de vie économique, de formation psychique »(Billoux 1951 : 22).

Dans la réalité, on voit que cette conception est interprétée de manière très arbitraire et souple : comment pourrait-on sinon qualifier une langue de stable (par exemple l'allemand ou le catalan) et une autre d'instable et point nationale (comme ce fut le cas de l'occitan). En tous cas, la prise de position de Marty, mais plus encore la négligence envers la langue et la culture occitane exprimée par la loi Marty (1948), fut accueillie avec déception par les militants ${ }^{23}$ sensibles aux intérêts de la langue, comme le montre la réaction de Max Allier, poète occitan et ancien membre de la Résistance :

Le PCF, selon une tradition déjà ancienne, paraissait accepter le principe de cette revendication occitane et la favoriser. Et l'on put croire que la partie était sur le point d'être gagnée quand on apprit qu'André Marty et le groupe communiste préparaient un projet de loi en faveur de l'enseignement des langues dites minoritaires dans le cadre national. La déception survint alors et brutale. Ce fut à ce moment en effet, que parut la brochure de Staline sur les questions linguistiques. Elle avait certes dû être préparée par des spécialistes au fait de la question et portait des jugements dont la valeur demeure. Mais partant de la situation en URSS, elle comportait aussi une affirmation qui retentit aux oreilles des Occitans comme le chant funèbre de leurs espérances. Staline n'y reconnaissait la légitimité d'une langue et d'une culture qu'autant qu'elles étaient l'expression d'une nation. Et le projet de loi d'André Marty, enfin déposé, prétendait reconnaitre aux seuls catalans le droit à leur culture, rejetant sans appel et sans explication les dialectes occitans...Ce fut une douche froide (Allier 1982 : 157).

En se conformant aux principes de Staline sur le sujet des nationalités, Marty sacrifia pour ainsi dire l'occitan. Quoi qu'il en soit, le PC avait manqué sa chance au sujet de l'occitan et la tâche de faire une place à l'occitan dans le cadre de la loi incombait donc à Deixonne, qui entre temps avait changé d'opinion. Même si les socialistes étaient majoritairement hostiles à toutes revendications linguistiques, ce fut une petite minorité autour de Félix Gouin (Bouches-du-Rhône) et François Tanguy-Prigent (Finistère) qui influença favorablement Deixonne. Ce dernier, très inspiré par Jean

\footnotetext{
23 Félix Castan dit à ce sujet : «Nous ne pensons pas que l'occitanisme constituait une des préoccupations majeures du Parti [...]. D'ailleurs, dans le Parti communiste, ça n'intéressait personne. Je ne pouvais même pas en parler sauf dans une période récente. J'avais l'air d'un fou, d'un simple fou » (Abrate 2001 : 432).
} 
Jaurès $^{24}$, prédécesseur dans son fief électoral d'Albi-Carmaux, contribua à son tour à faire entrer, d'après la recommandation de l'écrivain Max Rouquette (Institut d'études occitanes) ${ }^{25}$, le terme « occitan » dans le texte. Après avoir proposé l'appellation « langue d'oc », la Commission opta pour « provençal et languedocien» pour finalement inscrire « occitan»-c'est ainsi que fut officialisé la dénomination actuelle. En somme on s'aperçoit que les opinions et les perceptions des différentes langues régionales au sein du PCF sont fortement dépendantes des personnages particuliers. L'interprétation restrictive à la « française » de la conception stalinienne, cette dernière permettait au fond, du moins théoriquement, une promotion scolaire de l'occitan, sert de support pour exclure de manière arbitraire l'occitan.

L'abandon de l'occitan dans le discours de Marty reste tout de même obscur. Y aurait-il, à côté des préférences personnelles de Marty, des raisons politico-stratégiques? Pensait-il qu'il fallait se recentrer sur le catalan, langue parlée par un peuple en lutte contre le franquisme, et délaisser aux socialistes et aux autres partis le combat pour l'occitan, langue à laquelle on reprochait son manque d'unité linguistique, et aux locuteurs occitans auxquels on objectait l'absence d'une nationalité bien définie?

\section{Conclusion}

En somme, que dire de cet engagement communiste après la guerre? Les communistes français étaient confrontés à la difficulté d'harmoniser trois positions différentes : le patriotisme pour une République unie avec le français comme langue de la République, la conception marxiste-stalinienne et l'attachement aux langues et dialectes régionaux. Pour atteindre ce triple objectif, les députés communistes se présentèrent comme une force patriotique marquée par la résistance au nazisme et au franquisme. Une force indépendante qui peut dès lors revendiquer sans rougir la promotion des langues régionales, et tout cela en dépit du fait déplorable qu'une partie des associations linguistiques furent accusées d'avoir collaboré pendant la Seconde Guerre mondiale avec le régime de Vichy et l'occupant. Dans cette optique, il apparaissait même souhaitable de s'emparer à Paris des associations bretonnes, réputées réactionnaires, comme nous avons pu le voir plus avant.

${ }^{24} \mathrm{Il}$ faut ajouter que ce fut le poète Jòrgi Reboul (Félibrige/Marseille) qui expédia le texte pertinent de Jaurès avec le «manifeste de Belmont» à l'ancien chef du gouvernement provisoire Félix Gouin, qui le transmettra en suite à Deixonne. Voir Moliner (2010 : 203-204).

25 Voir aussi Moliner (2010 : 219-220). 
En même temps nous avons pu observer que le fait d'avoir interprété de manière très restrictive la doctrine stalinienne, comme le fit Marty en attribuant à la seule population de la région alsacienne un statut de minorité nationale, a certainement créé une source de contradictions et d'ambiguités. Le fait d'avoir récusé farouchement le fédéralisme et un régime bilingue, comme nous l'avons vu chez Hervé pour la Basse-Bretagne, ou le fait d'avoir rejeté le bilinguisme pour la Catalogne du nord tout en prônant la légitimité des aspirations antifranquistes et autonomistes en Catalogne du sud, souligne qu'il y avait au sein du PCF une tradition qu'on pourrait définir de « jacobine ».

Finalement, on peut aussi en déduire l'existence d'une perception linguistique qui ordonne les langues régionales hiérarchiquement : en première position nous trouvons les dialectes alsaciens et l'allemand standard, ses locuteurs sont classés comme une «minorité linguistique/nationale » vivants dans des rapports bilingues, en seconde position se situent le catalan et le breton qui sont considérés comme des «langues familiales », des «instruments de culture populaire » situés dans des régions à majorité francophone et disposant d'un passé national propre, mais désormais révolu. L'occitan, divisé par ses dialectes, se retrouve en troisième position. L'histoire de ses locuteurs est liée très tôt à celle de la France, donc il n'y a jamais eu de nationalité occitane. Ceci s'applique aussi aux locuteurs d'autres langues oubliées telles que le flamand, le basque ${ }^{26}$ ou le corse. Dans cette perspective il est explicable qu'il n'y eut à notre connaissance aucun député PCF qui prit la parole pour le basque ou le flamand dans la période allant de 1937 à 1951. Il est aussi hautement significatif que les locuteurs corses et militants au sein du PC s'abstinrent de faire valoir leurs propres revendications durant les négociations dans le cadre de la loi Deixonne ${ }^{27}$.

En fin de compte il est intéressant de comparer l'engagement parlementaire du PCF avec celui des autres partis/groupes parlementaires afin d'en mesurer l'impact. Certainement au parlement il n'y eut nul autre parti qui allât aussi loin au sujet d'une autonomie politique et culturelle au sujet de l'Alsace. Quoi qu'en veuille reprocher aux communistes, ils surent prendre dès 1936, donc très tôt, des décisions avec l'appui collectif du groupe en faveur des langues régionales. Les autres députés et supporters des langues régionales, les radicaux et les royalistes/conservateurs, combattaient en général en ordre dispersé, souvent isolés dans leurs propre partis, une exception fut le Parti

\footnotetext{
${ }^{26}$ L'insertion tardive, en 1949, de la langue basque dans le cadre de loi Deixonne est due à l'activité tenace de Jean Errécart, député MRP du département Basses-Pyrénées (Moliner 2010 : 217).

27 Le procès verbal mentionne le député communiste d'Ajaccio, Arthur Giovoni, lors du débat au sujet de la future loi Deixonne lors de la séance du 16 février 1949 à la Commission de l'Éducation Nationale : « M. Giovoni, qui pourrait cependant formuler des revendications à propos du corse, déclare vouloir s'en tenir uniquement au problème du breton. » (Moliner 2010 : 199)
} 
démocrate populaire (PDP) avec le vétéran Pierre Trémintin et plus tard le MRP. À la sortie de la Seconde guerre mondiale cette tendance pour une promotion des langues régionales se renforça chez les élus nationaux du PCF : les députés bretons furent les premiers à déposer un projet (1947). Un second projet en faveur du catalan est déposé en (1948). Pour obtenir gain de cause ils s'allièrent même avec des sénateurs du MRP/RPF (1948 et 1949). Paradoxalement le PCF n'a jamais pu tirer un bénéfice politique de son engagement parlementaire, c'est le député Maurice Deixonne (SFIO/Tarn), uns des pires ennemis des communistes, qui eut finalement le soin de proposer une loi-cadre globalement favorable aux langues régionales, adoptée finalement en janvier 1951 par l'AN.

\section{Références bibliographiques}

Abrate, Laurent. 2001. Occitanie 1900/1968. Des idées et des hommes: L'émergence et l'bistoire de la revendication occitane. Puylaurens : Institut d'Estudis Occitans.

Antoine, Gérald et Bernard Cerquiglini. 2000. Histoire de la langue française. Paris : CNRS.

Ar Falz. 1946. Le manifeste de Ker-Vreiz. Ar Falz 2 (1 ${ }^{\text {er }}$ trimestre).

Allier, Max. 1982. Témoignage d'un poète occitan. Cabiers d'Histoire de l'IRM 9 : 155-158.

Billoux, François. 1950a. Les grandes responsabilités des communistes d'Alsace et Lorraine. Cabiers du communisme $3: 35-47$.

Billoux, François. 1950b. Sur certaines particularités nationales en Alsace et Lorraine. Cabiers du communisme $6: 52-55$.

Billoux, François. 1951. La politique allemande de paix de l'union soviétique. Les conférences éducatives du parti communiste français $2: 1-32$.

Cadé, Michel. 1998. Études d'bistoire sociale, politique et des représentations : Synthèse de travaux en vue d'obtenir l'habilitation à diriger des recherches. Perpignan : Université de Perpignan.

Calvé, Armel. 1994. Histoire des Bretons à Paris. Spezet : Coop Breizh.

Calvet, Louis-Jean. 1974. Linguistique et colonialisme. Paris : Payot.

Chanet, Jean-François. 1996. L'école républicaine et les petites patries. Paris : Aubier.

Circulaire Ripert Instructions du 9 octobre 1940 de M. le Secrétaire d'État à l'I. P. et la Jeunesse. 1940 Bulletin Départemental de l'Instruction primaire, Académie d'Aix, département de Vaucluse: 299-301.

Courouau, Jean-François. 2008. Mon lengatge bèl: Les choix linguistiques minoritaires en France (1490-1660). Genève : Droz.

Décret Marie. Ministère de l'Éducation nationale (Ministre André Marie). N52-1347 du 18 décembre 1952.

Desgranges, Abbé. 1960. Journal d’un prêtre député 1936-1940. Paris/Genève : La Palatine. 
Forest, Philippe, dir. 1991. Qu'est-ce qu'une Nation? Texte intégral de E. Renan. Paris : Bordas.

Giacomo, Mathée. 1975. Les propositions communistes, la loi Deixonne et les langues régionales. La nouvelle critique $88: 27-30$.

Guiomar, Jean-Yves. 1970. Régionalisme, fédéralisme et minorités nationales en France entre 1919 et 1939. Le Mouvement social $70: 89-108$.

Keravel, Armand. 1947. La Proposition Pierre Hervé. Ar Falz 10 (juin).

Keravel, Armand. 1970. Tanguy Prigent, Breton et bretonnant. Allocution diffusée par Radio-Brest, janvier 1970. Dans I. Ti Kaled, sous la direction de Mireille Prigent et Tanguy Priget, 313-315. Paris : Club socialiste du livre.

Lafont, Robert. 1974. La revendication occitane. Paris : Flammarion.

Le Bail, Albert. 1948. Groupe des instituteurs et professeurs laïques bretons: Demande de subvention. Dans Rapport du Préfet et de la Commission départementale : Procès verbaux des délibérations. Première session ordinaire de 1948, 133-140. $2^{\mathrm{e}}$ séance 12 mai. Quimper : Menèze.

Lebsanft, Franz. 2004. Frankreichs Mehrsprachigkeit. Dans Literatur und Mehrsprachigkeiton, sous la direction de Monika Schmitz-Emans, 175-188. Heidelberg : Synchron.

Lenin, Vladimir, Iosif Stalin et Nikolai Boukharin. 1927. Le Communisme et la question nationale et coloniale par Lénine, Staline et Boukharine. Paris : Bureau d'éditions, de diffusion et de publicité.

Loi Trémintin [LT]. Documents parlementaires [DP] Annexe (N¹41. 1936 : 768-769). Séance du 5 juin 1936.

Loi Dahlet [LD]. DP Annexe (N921. 1936 : 1560-1561). $3^{\text {e }}$ Séance du 30 juillet 1936.

Loi Marty [LM]. DP Annexe (N5028. 1948 : 1-6). Séance du 24 juillet 1948.

Loi Vourc’h [LV]. DP Annexe (N5071. 1948 : 1-14). Séance du 27 juillet 1948.

Lougal, Pierre [Pseudonyme de Joseph d'Arbaud]. 1925. La langue d'oc et l'enseignement. Le Feu 2 : 68.

Martel, Philippe. 1990. Autour de la loi Deixonne. Dans Actes de l'université d'été (Universitat occitana d'estiu) 1990, sous la direction d'Agnès Lobier, 41-57. Nîmes : MARPOC.

Martel, Philippe. 2001. La France et l'occitan à l'époque contemporaine: Histoire d'une étrange politique linguistique. Dans Dix siècles d'usages et d'images de l'occitan, sous la direction Henri Boyer et Philippe Gardy, 367-383. Paris : L'Harmattan.

Marty, André. 1952. Il n'y a pas de "langue occitane »: Une réponse d'André Marty à M. Henri Lefebvre. Patriote de Toulouse. Toulouse : 16 février.

Maugué, Pierre. 1970. Le particularisme alsacien. 1918-1967. Paris : Presses d'Europe.

Moliner, Olivier. 2010. Frankreichs Regionalsprachen im Parlament. [Beihefte zu Quo Vadis, Romania 36]. Wien : Praesens.

Monzie, Anatole de. 1925. Circulaire du 14 août 1925 relative aux idiomes locaux. Bulletin départemental de l'Éducation Nationale. Inspection académique. Morbihan : $1^{\mathrm{er}}$ septembre 1925.

Nouvet, Antoine. 2001. La loi Deixonne et les débats sur l'enseignement des langues régionales. Dans La vie politique française 1945-1953. Paris I : Centre d'histoire sociale du XX siècle. 
Paul, Gabriel. 2007a. Lettre manuscrite à l'auteur 21 novembre.

Paul, Gabriel. 2007b. Lettre manuscrite à l'auteur du 10 décembre.

Rapport Desgranges [RD]. DP Annexe (N²732. 1937 : 916-919). Séance du $1^{\text {er }}$ juillet 1937.

Reimeringer, Bernard. 1977. Un communisme régionaliste? Dans Régions et régionalisme en France du XVIII siècle à nos jours, sous la direction de Christian Gras et Georges Livet, 361-392. Paris : PUF.

Résolution Hervé [RH]. Annexe (Nº1326. 1947 : 1-6). 2e séance du 16 mai 1947.

Résolution Lempereur [RL]. DP Annexe (N¹641. 1947 : 1-3). 2e séance du 12 juin 1947.

Résolution Rosenblatt/Schell [RRS]. DP Annexe (N¹0354. 1950 : 1). Séance du 19 juin 1950.

Sagnes, Jean. 1982. Le PCF et la culture occitane. Cabiers d'Histoire de l'IRM 9 : 1-190.

Toulemont, Léon. 1947. Les conférences de Ker-Vreiz. La Bretagne à Paris 24, 11 avril : 1.

Trabant, Jürgen. 1981. Die Sprache der Freiheit und ihre Feinde. Zeitschrift für Literaturwissenschaft und Linguistik 41 : 70-89. 